\title{
Simulation Study for Energy Harvesting Using Polyvinylidene Fluoride flag in the Flow
}

\author{
Lv Fengchi ${ }^{1, a}$, Song Rujun ${ }^{1, b}$, Zhang Xingxu ${ }^{1, c}$, Shan Xiaobiao ${ }^{1, d}$, Xie Tao ${ }^{1, e^{*}}$ \\ ${ }^{1}$ School of Mechatronics Engineering, Harbin Institute of Technology, 92 West Dazhi Street, \\ Nan Gang Distric, Harbin, 150001, China \\ alvfengshichi@163.com, bsongrujunok@126.com, 'xingxuz@163.com, 'shanxiaobiao@hit.edu.cn, \\ exietao@hit.edu.cn
}

Keywords: flow, piezoelectric, energy harvesting, simulation

Abstract: In this paper, Dynamic response and energy harvesting potential of single piezoelectric flag in the uniform flow is analyzed through $2 \mathrm{D}$ simulation method. A piezoelectric material named Polyvinylidene Fluoride (PVDF) is used to manufacture the piezoelectric flag. Special example is introduced to analyze the dynamic response and energy harvesting potential in the axial flow. The generated electric voltage and special points' displacement variation with time are presented. The harvesting energy performance is researched. It is found that the flapping of the PVDF flag comes into limit-cycle after a few cycles adjustment and five characteristic points flap with the same cycle at the velocity of $3 \mathrm{~m} / \mathrm{s}$. the optimal resistance has an inverse proportion relationship with the velocity. With the increase of the inlet velocity the voltage will reach a stable value while the maximum output power will increase persistently. This paper will help to find more efficient energy harvester, and the energy harvester will be able to supply energy for the MEMS in the flow.

\section{Introduction}

The flow induced vibration exists everywhere. When the wind comes, the flags flap and the leaves float. And the waterweeds flutter if the water flows. Each of them represents the phenomena of fluid-flexible-structure interaction. The development of low energy-consuming electronics urgently needs sustainable power generator instead of battery in some special environmental conditions such as the remote region, the dangerous region which is inaptitude for battery supplying energy. An idea comes into mind that we can convert flow induced vibration energy to electric energy by a sustainable energy generator. Piezoelectric flag is such a device that can generate electricity from flow induced vibration.

Recently, many researchers have done research on the flow-induced vibration to harvest energy. A practical eel structure using PVDF piezoelectric material was developed as a small subsurface ocean/river power generator by Taylor[1]. Energy harvesting from unsteady turbulent vortex shedding using piezoelectric flags located behind bluff bodies was investigated through numerical simulations and experiments[2-6], which improved the converting efficiency by attaching a fin to the tail of the cantilever beams $[3,4,7,8]$. In axial flow, energy harvesting from the fluttering piezoelectric flag was analyzed by numerical simulation [9-14]. Dunnmon[15] studied the nonlinear limit-cycle oscillations of energy harvester to enhance generating power performance from aerodynamic flows. Furthermore, in order to improve power generation ability, some scholars presented some meaningful structures. Kwon presented a T-shaped piezoelectric cantilever to adapt to the axial flow, A bistable energy harvester using turbulent wind was leaned by Kitio Kwuimy.

In this paper, simulation study for energy harvesting using flexible piezoelectric flag in axial flow is presented. This study uses immersed boundary-lattice Boltzmann method coupling with Euler-Bernoulli beam and piezoelectric theory. In section I, the physical mode and numerical method are researched. The validity is verified in section II and hydroelastic response and energy harvesting ability of single flexible piezoelectric flag are analyzed by coupling numerical method in section III. Conclusion shows in section IV. 


\section{Problem statement and numerical method}

In this section, Physical mode is manufactured. Fig. 1 shows a schematic of energy harvesting piezoelectric flag and an equivalent circuit.

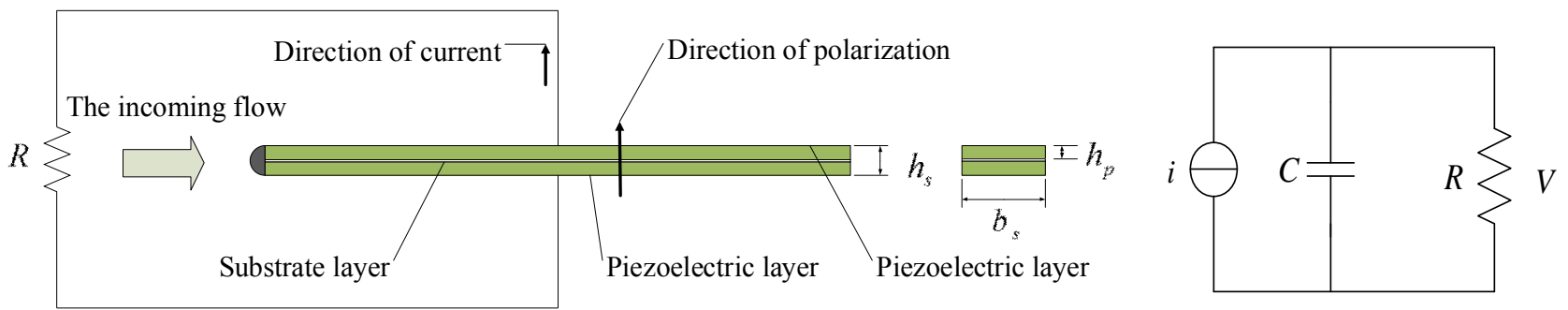

a) Schematic of flexible piezoelectric flag with the continuous electrodes

b) Schematic of equivalent circuit of the piezoelectric flag

Fig. 1 Schematic of the flexible piezoelectric flag with continuous electrodes and the equivalent circuit

In Fig. 1(a), the energy generator consists of two outer continuous piezoelectric layers adhered to an inner substrate layer. The thickness of piezoelectric layers is $h_{p}$ and the total thickness of the flag is $h_{s}$. From the equivalent circuit in Fig. 1(b), $i$ is the transient current, $V$ is the transient voltage, $C$ is the equivalent capacitance of the piezoelectric flag. The flexible flag is immersed into an axial, uniform flow. Table 1 lists the governing parameters of the generator. Some assumptions are made to simplify the model: the thickness of the substrate layer is much thinner than the piezoelectric layer and the flag is inextensibility in length direction. $h$ is considered to be $2 h_{p}$, and the bending stiffness $K_{b}$ is considered by ignoring the axial deformation.

Table 1 Critical dimensional parameters of the energy harvesting system

\begin{tabular}{lll}
\hline Parameters & definition & $\mathrm{unit}$ \\
\hline$L_{s}$ & The flag length & $\mathrm{m}$ \\
$h_{s}$ & The total flag thickness & $\mathrm{m}$ \\
$b_{s}$ & The flag width & $\mathrm{m}$ \\
$K_{b}$ & The flag bending stiffness & $\mathrm{N} / \mathrm{m}$ \\
$K_{s}$ & The stretching coefficient & \\
$\rho_{s}$ & The flag density & $\mathrm{Kg} / \mathrm{m}^{3}$ \\
$U_{f}$ & The incoming flow speed & $\mathrm{m} / \mathrm{s}$ \\
$\rho_{f}$ & The incoming flow density & $\mathrm{Kg} / \mathrm{m}^{3}$ \\
$\mu_{f}$ & The incoming flow dynamic viscosity & $\mathrm{Pa} \cdot \mathrm{s}$ \\
$\varepsilon$ & The piezoelectric electric permittivity & $\mathrm{F} / \mathrm{m}$ \\
$R$ & The electric circuit resistance & $\Omega$ \\
$e_{31}$ & The piezoelectric stress constant & $\mathrm{C} / \mathrm{m}^{2}$ \\
\hline
\end{tabular}

In simulation, some characteristic scales are introduced: $L_{s}$ for length, $U_{f}$ for velocity, $L_{s} / U_{f}$ for time, $\rho_{f} U_{f}^{2}$ for the force $\boldsymbol{F}, \rho_{f} U_{f}^{2} L_{s}$ for the tension force $\boldsymbol{T}$. The Navier-Stokes equations are introduced to describe the laminar, viscous, Newtonian and incompressible flow and the nondimensional forms are given by

$$
\frac{\partial \boldsymbol{u}}{\partial t}=-\nabla P+\frac{1}{R e} \nabla^{2} \boldsymbol{u}+\boldsymbol{F}_{f} \quad \nabla \cdot \boldsymbol{u}=0
$$

where, $\boldsymbol{u}=(\mu, v)$ is the inlet velocity, $P$ is the pressure, $R e=\rho_{f} L_{s} U_{f} / \mu_{f}$ is the Reynolds number, $\boldsymbol{F}_{f}$ is the body force. Here, the piezoelectric flags are supposed to be large elastic deformation with small thickness, negligible the extension in the length direction and structural damping.

According to the physical mode, the structure governed equation can be presented in Eq. (2) and Eq. (3)

$$
m_{s} \frac{\partial^{2} \boldsymbol{X}}{\partial t^{2}}=\boldsymbol{T} \frac{\partial^{2} \boldsymbol{X}}{\partial s^{2}}-K_{b} \frac{\partial^{4} \boldsymbol{X}}{\partial s^{4}}+\frac{\Theta V}{b} \frac{d}{d s}[\delta(s)-\delta(s-L)]-\boldsymbol{F}
$$




$$
\begin{aligned}
& C \frac{\partial V}{\partial t}+\frac{V}{R}+\Theta \int_{0}^{L} \frac{\partial^{3} X}{\partial s^{2} \partial t} d s=0 \\
& P=\frac{1}{T} \int_{0}^{T} \frac{V^{2}}{R} d t
\end{aligned}
$$

where, $s$ is the Lagrangian coordinate along the length, $X$ is the position vector of a point on the piezoelectric flag, $m_{s}=\rho_{s} h_{s}$ is the mass per unit length of the flag, $T$ is the tension force determined by the constraint inextensibility, $K_{b}=E h_{s}{ }^{3} / 12$ is the bending rigidity, $\Theta=e_{31} h_{s} b_{s} / 4$ is the piezoelectric coupling term, $V$ is the voltage across the electrode, $\delta$ is a Dirac-Delta function, $C=\varepsilon L_{s} b_{s} / h_{s}$ is the equivalent capacitance of the piezoelectric flag, $R$ is the external resistor. $V$ and $P$ are the output voltage and power. $T$ is the flapping cycle. Where, $\rho_{s}, e_{31}, \varepsilon, L_{s}, h_{s}, b_{s}$ are in Table 1.

\section{Validation of the numerical method}

In order to verify the validity of the numerical method in energy harvesting fleid, the comparison with Akcabay's work is carried out with energy harvesting ability. In this simulation, the parameters of $\beta_{\rho}=10, \beta_{U}=6, v q=5.3, q=1 / 646, q_{p}=1 / 2, R \mathrm{e}=210$ when $R^{\prime} b^{\prime}$ varies $30 \Omega \cdot \mathrm{m}, 300 \Omega \cdot \mathrm{m}, 3000 \Omega \cdot \mathrm{m}$ and 3000 $\Omega \cdot \mathrm{m}$. Further information of the meaning of the parameters could be found in Refs.

Table 2 Comparison of numerical simulation result with that of Akcabay for single piezoelectric flag in voltage and power density

\begin{tabular}{ccccc}
\hline$R^{\prime} b^{\prime}(\Omega \cdot \mathrm{m})$ & $\begin{array}{c}\text { Voltage of this } \\
\text { paper } V^{\prime}(\mathrm{V})\end{array}$ & $\begin{array}{c}\text { Power density } \\
\text { of this paper } \\
V^{\prime 2} / R^{\prime} b^{\prime}(\mathrm{W} / \mathrm{m})\end{array}$ & $\begin{array}{c}\text { Voltage of } \\
\text { Akcabay } V^{\prime}(\mathrm{V})\end{array}$ & $\begin{array}{c}\text { Power density } \\
\text { of Akcabay } \\
V^{\prime 2} / R^{\prime} b^{\prime}(\mathrm{W} / \mathrm{m})\end{array}$ \\
\hline 30 & 3.53 & 4.167 & 3.55 & 4.2 \\
300 & 14.85 & 7.35 & 15.5 & 8.0 \\
3000 & 17.68 & 10.42 & 18.2 & 11 \\
15000 & 17.3 & 0.199 & 18 & 0.2 \\
\hline
\end{tabular}

Table 2 shows the numerical results of comparison with Akcabay's in the voltage and power density, it can be seen that our simulations are good agreement with the work of Akcabay, which means the validity of our numerical method in energy harvesting ability.

\section{Dynamic response and energy harvesting potential of single piezoelectric flag}

PVDF (Polyvinylidene Fluoride) which is chosen as the piezoelectric material is flexible enough to meet the request of flow induced, whose density $\rho_{s}$ is $1700 \mathrm{~kg} / \mathrm{m}^{3}$. its piezoelectric electric permittivity $e_{31}$ is $-0.06 \mathrm{c} / \mathrm{m}^{2}$, the elasticity modulus $E$ is $3 \mathrm{GPa}$. The flag is immersed in the air whose density $\rho_{f}$ is $1.209 \mathrm{~kg} / \mathrm{m}^{3}$. The parameters of the flag is that $L_{s}=100 \mathrm{~mm}, b_{s}=10 \mathrm{~mm}, h_{s}=0.05 \mathrm{~mm}$. The velocity varies from $1 \mathrm{~m} / \mathrm{s}$ to $10 \mathrm{~m} / \mathrm{s}$, which is in the limit-cycle domain.

The velocity of $3 \mathrm{~m} / \mathrm{s}$ and the resistance of $3 \mathrm{M} \Omega$ are chosen as the example to investigate the dynamic response of the single flag. Fig. 2 shows the vibration state of the flag at the velocity of $3 \mathrm{~m} / \mathrm{s}$. In Fig. (a) and Fig. (b), Results show that the flapping comes into limit-cycle after a few cycles adjustment and five characteristic points flap with the same cycle; the output electric is alternating synchronized with the same time cycle T of the flapping of flag. In Fig. (c) and Fig. (d), Results show that the flapping curve of tail comes into view as the number ' 8 ', which is coincide with Zhang's experiment result and the phase of the tail appears to be roundness, which means the flapping of tail is similar to simple harmonic oscillation. 


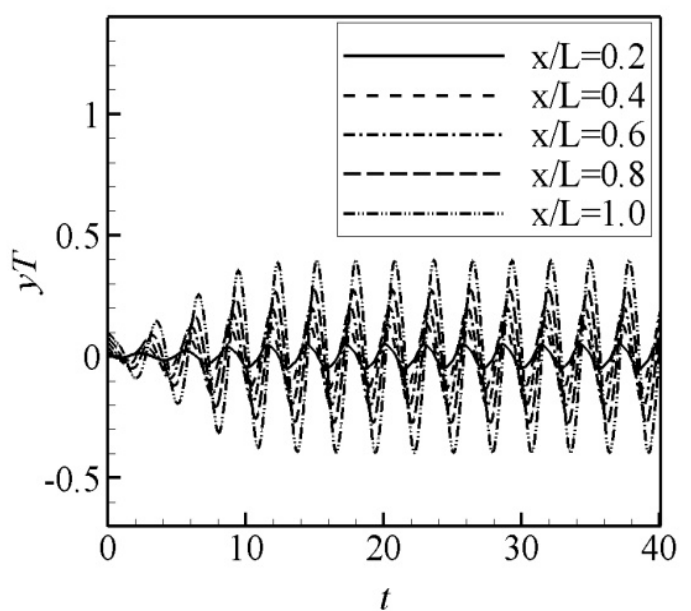

(a) Time histories of the five points of the flag

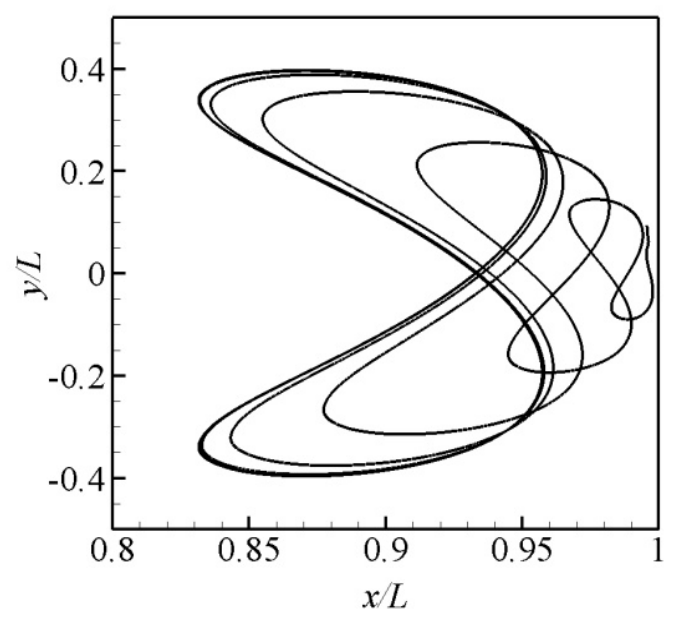

(c) fluttering curve of the tail

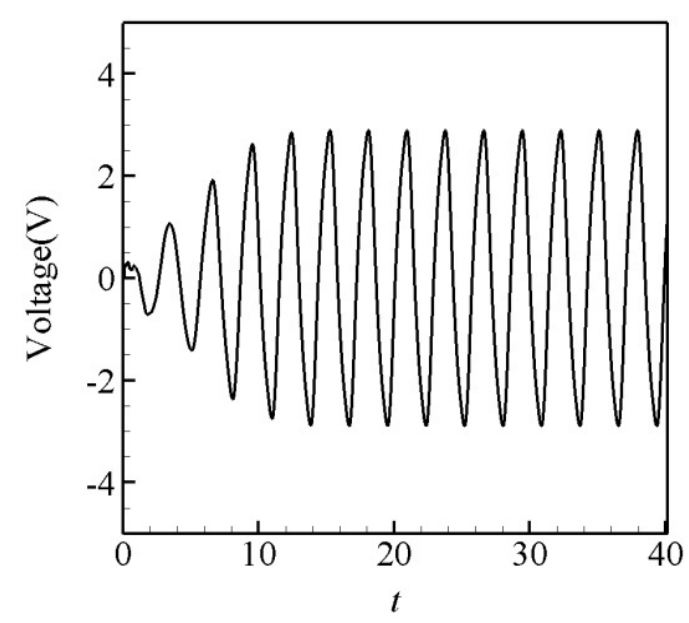

(b) Time histories of the generated electric voltage

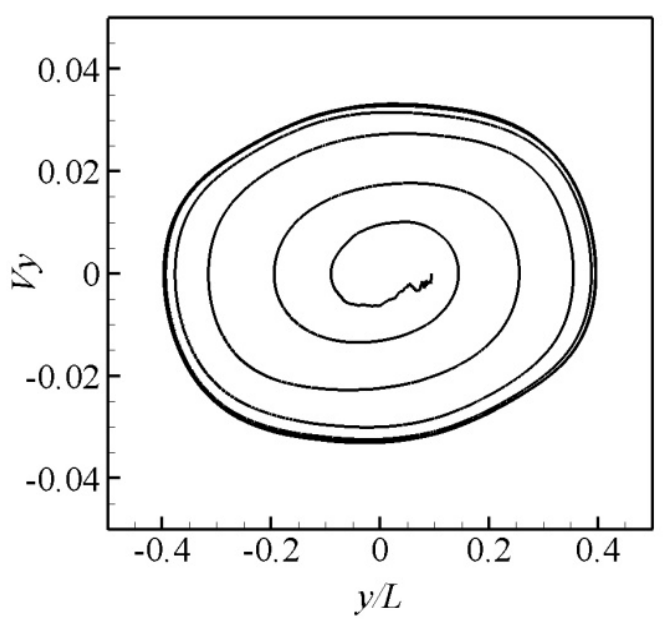

(d) Phase of the tail

Fig. 2 Vibration state and characters of the flag at the velocity of $3 \mathrm{~m} / \mathrm{s}$

Fig. 3(a) shows the Optimal resistance changing by the velocity. The Optimal resistance has an inverse proportion relationship with the velocity. That is

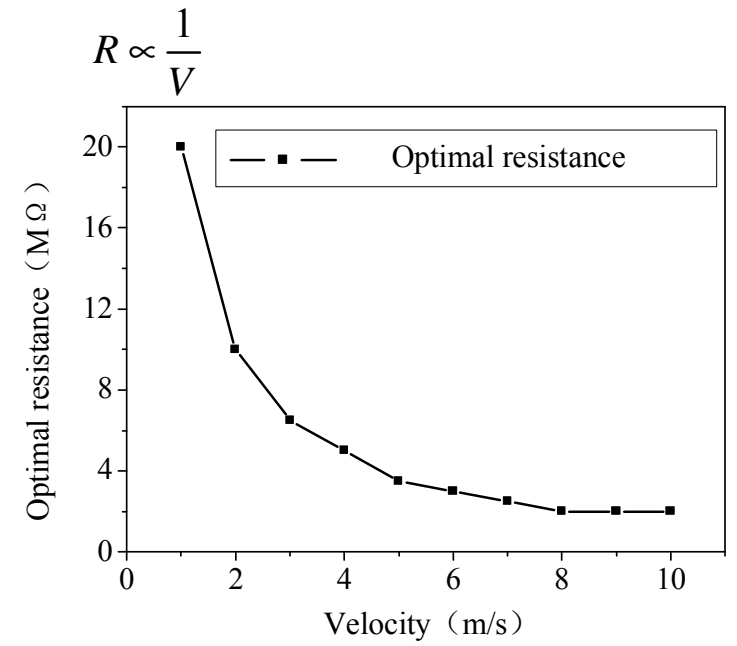

(a) Optimal resistance changing by inlet velocity

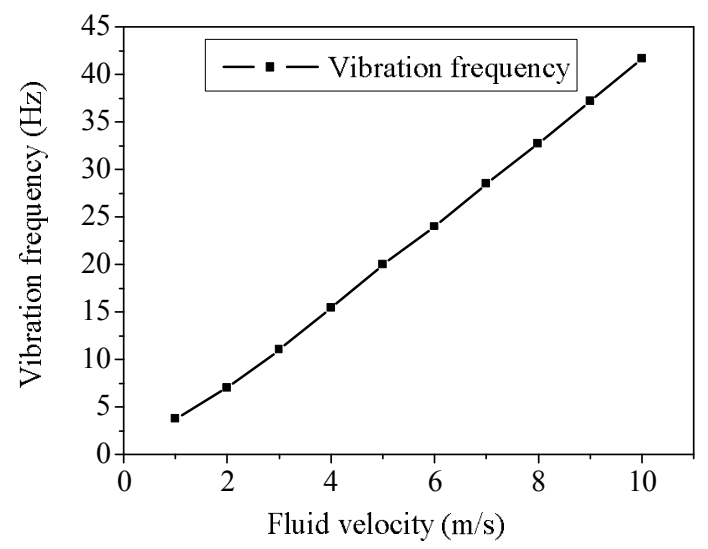

(b) Vortex street shedding frequency changing by inlet velocity

Fig. 3 Optimal resistance changing by inlet velocity

Fig. 3(b) shows the Vortex street shedding frequency changing by inlet velocity. The frequency is 
in direct proportion to the velocity. That is

$f \propto V$

Now we can get

$R \propto \frac{1}{f}$

$R=\frac{k}{f}$

The resistance is in inverse proportion to the vortex street shedding frequency.

The maximum output power is related with both the optimal resistance and the circuit voltage on both ends.

$P=\frac{U^{2}}{R}=\frac{U^{2}}{\left(\frac{k}{f}\right)}=\frac{U^{2} f}{k}=\frac{U^{2} V}{k_{1}}$

When the inlet velocity increases, the vortex street shedding frequency that makes major contribution to the vibration frequency of the harvester rises, causing the optimal resistance increasing. The maximum output power is decided by both the optimal resistance and the circuit voltage. We cannot get the real value of the maximum output power only by the circuit voltage because the optimal resistance is variant with the velocity. That is to say that keeping the voltage stable the maximum output power is in direct proportion with the inlet velocity. Fig. 6 shows the relationship between both the maximum output power and circuit voltage and different inlet velocities. In the beginning both the circuit voltage and the maximum output power increases with the inlet velocity quickly. When the velocity attains $4 \mathrm{~m} / \mathrm{s}$ the circuit voltage is tending towards stability while the maximum output power increases continuously. The maximum output power is approximately linear relationship with the velocity as the circuit voltage changes only a little. In conclusion, with the increasing of the inlet velocity the voltage will reach a stable value while the maximum output power will increase persistently.

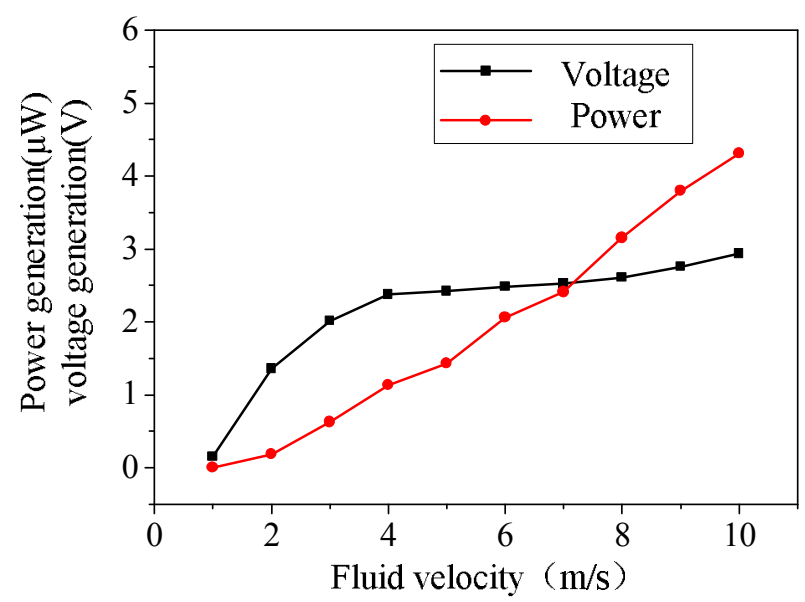

Fig. 4 Power and voltage generation with optimal resistance changing by inlet velocity

\section{Conclusion}

In this paper, Dynamic response and energy harvesting potential of single piezoelectric flag in the uniform flow is analyzed. A piezoelectric material named PVDF is used to manufacture the piezoelectric flag. Special example is introduced to analyze the dynamic response and energy harvesting potential in the axial flow. The generated electric voltage and special points displacement variation with time are presented. The harvesting energy performance is researched.

If the inlet velocity is $3 \mathrm{~m} / \mathrm{s}$, the flapping of the PVDF flag comes into limit-cycle after a few cycles 
adjustment and five characteristic points flap with the same cycle. The output electric is alternating synchronized with the same time cycle $T$ of the flapping of flag. The flapping curve of tail comes into view as the number ' 8 '.

The optimal resistance has an inverse proportion relationship with the velocity which is in direct proportion to the flapping frequency of the PVDF flag. The maximum output power is related with both the optimal resistance and the circuit voltage on both ends. When the velocity attains $4 \mathrm{~m} / \mathrm{s}$ the circuit voltage is tending towards stability while the maximum output power increases continuously. The maximum output power is approximately linear relationship with the velocity as the circuit voltage changes only a little.

This paper will help to find more efficient energy harvester, and the energy harvester will be able to supply energy for the MEMS in the flow.

\section{Acknowledgment}

This work was financially supported by the Fundamental Research Funds for the Central Universities (Grant No. HIT. NSRIF. 2014059 and No. HIT. KISTP. 201412).

\section{Reference}

[1] Taylor, G.W., et al., The Energy Harvesting Eel: a small subsurface ocean/river power generator. IEEE Journal of Oceanic Engineering, 2001. 26(4): p. 539-547.

[2] Akaydin, H.D., N. Elvin, and Y. Andreopoulos, Energy Harvesting from Highly Unsteady Fluid Flows using Piezoelectric Materials. Journal of Intelligent Material Systems and Structures, 2010. 21(13): p. 1263-1278.

[3] Akaydin, H.D., N. Elvin, and Y. Andreopoulos, The performance of a self-excited fluidic energy harvester. Smart Materials and Structures, 2012. 21(2): p. 025007.

[4] Weinstein, L.A., et al., Vortex shedding induced energy harvesting from piezoelectric materials in heating, ventilation and air conditioning flows. Smart Materials and Structures, 2012. 21(4): p. 045003.

[5] Akaydın, H.D., N. Elvin, and Y. Andreopoulos, Wake of a cylinder: a paradigm for energy harvesting with piezoelectric materials. Experiments in Fluids, 2010. 49(1): p. 291-304.

[6] Ahmadian, M., et al., Generation of electrical energy using short piezoelectric cantilevers in flowing media. The 16th International Symposium on: Smart Structures and Materials \& Nondestructive Evaluation and Health Monitoring. International Society for Optics and Photonics, 2009: p. 728807-728807-8.

[7] Sirohi, J. and R. Mahadik, Piezoelectric wind energy harvester for low-power sensors. Journal of Intelligent Material Systems and Structures, 2011. 22(18): p. 2215-2228.

[8] Li, S., J. Yuan, and H. Lipson, Ambient wind energy harvesting using cross-flowfluttering. Journal of Applied Physics, 2011. 109(2): p. 026104.

[9] Doaré, O. and S. Michelin, Piezoelectric coupling in energy-harvesting fluttering flexible plates: linear stability analysis and conversion efficiency. Journal of Fluids and Structures, 2011. 27(8): p. 1357-1375.

[10] Shi, S., T.H. New, and Y. Liu, Flapping dynamics of a low aspect-ratio energy-harvesting membrane immersed in a square cylinder wake. Experimental Thermal and Fluid Science, 2013. 46: p. 151-161.

[11] Singh, K., S. Michelin, and E. De Langre, The effect of non-uniform damping on flutter in axial flow and energy-harvesting strategies. Proceedings of the Royal Society A: Mathematical, Physical and Engineering Sciences, 2012. 468(2147): p. 3620-3635.

[12] Tang, L., M.P. Païdoussis, and J. Jiang, Cantilevered flexible plates in axial flow: Energy transfer and the concept of flutter-mill. Journal of Sound and Vibration, 2009. 326(1-2): p. 263-276.

[13] Singh, K., S. Michelin, and E. de Langre, Energy harvesting from axial fluid-elastic instabilities 
of a cylinder. Journal of Fluids and Structures, 2012. 30: p. 159-172.

[14] Yadykin, Y., V. Tenetov, and D. Levin, The Flow-Induced Vibration of a Flexible Strip Hanging Vertically in a Parallel Flow Part 1: Temporal Aeroelastic Instability. Journal of Fluids and Structures, 2001. 15(8): p. 1167-1185.

[15] Dunnmon, J.A., et al., Power extraction from aeroelastic limit cycle oscillations. Journal of Fluids and Structures, 2011. 27(8): p. 1182-1198. 